\title{
Terapia de aceptación y compromiso de grupo: experiencia en un servicio público de salud mental
}

\author{
Therapy of group acceptance and commitment: experience in a public \\ service of mental health
}

\author{
Juan José Ruiz Sánchez ${ }^{\text {* }}$ \\ Unidad de Salud Mental Comunitaria de Úbeda \\ Recibido: $09-05-17$
}

\author{
Francisco Trillo Padilla² \\ Universidad de Almería
}

Aceptado: $01-06-17$

\section{Resumen}

Se presenta un resumen de las tres generaciones de la terapia de conducta, destacando en la tercera el papel de las funciones de la conducta en su contexto (especialmente del lenguaje). Se plantea los fundamentos de las Terapias Contextuales Grupales en base a su eficacia y eficiencia habitualmente en servicios públicos de atención psicológica. Se describe los focos de trabajo fundamentales de la Terapia de Aceptación y Compromiso de Grupo en referencia a discriminar como forma de derivar respuestas hacia acciones valiosas con sentido en la vida de las personas y por último se presenta de manera resumida nuestra experiencia de trabajo con la Terapia de Aceptación y Compromiso de Grupo con problemas ansiosos-depresivos en nuestro contexto de atención de salud mental pública.

Palabras clave: Terapias de grupo contextuales funcionales, eficacia, eficiencia, grupo, metáforas.

\begin{abstract}
It presents a summary of the fundamental aspects of the three generations of behavioral therapy. Third generation therapy is highlighted, the role of behavioral functions in their context (especially language). It raises the empirical foundations and applications of the Group Contextual Therapies based on its effectiveness and efficiency usually in public services of psychological attention. The main work focuses on Group Acceptance and Commitment Therapy and in reference to "realizing" or discriminating as a way of deriving responses to meaningful actions with meaning in people's lives and finally summarized our experience of how we work with Acceptance and Commitment Therapy Group to anxiety-depressive problems in our context of public mental health care.
\end{abstract}

Keywords: Functional contextual group therapies, efficacy, efficiency, group, metaphor.

\footnotetext{
*Autor para correspondencia

1. Psicólogo Clínico Especialista. Unidad de Salud Mental Comunitaria de Úbeda del S.A.S Jaén. España. Email: perotemix77@gmail.com

2. Psicólogo General Sanitario e Investigador de la Universidad de Almería, España.

Email: ftp699@inlumine.ual.es, chescotrillo@gmail.com
}

(C) Los autores. Este artículo es publicado por la Revista de Investigación en Psicología de la Facultad de Psicología, Universidad Nacional Mayor de San Marcos. Este es un artículo de acceso abierto, distribuido bajo los términos de la licencia Creative Commons Atribucion - No Comercia_Compartir Igual 4.0 Internacional. (http://creativecommons.org/licenses/by-nc-sa/4.0/) que permite el uso no comercial, distribución y reproducción en cualquier medio, siempre que la obra original sea debidamente citada. 


\section{LAS TRES GENERACIONES DE LA TERAPIA CONDUCTUAL}

Para llegar a comprender y entender el sentido de este artículo donde se pretende ahondar en las nuevas vías de investigación que se están abriendo en intervención grupal mediante las terapias de tercera generación, se presenta a continuación una pequeña reseña histórica sobre la evolución de las diferentes teorías psicológicas a lo largo del último siglo. De esta forma se pretende llegar a comprender el porqué de la aparición de nuevas teorías que abordan, a partir de los conocimientos que tenemos, un cambio en la función de la conducta.

\section{Primera Generación: La era Conductista (1913-1970)}

Tras el desarrollo a nivel teórico de las leyes del condicionamiento clásico de Pavlov y la formulación de la ley del efecto de Thorndike durante el final del siglo XIX y la primera década del Siglo XX, se empieza a constituir el marco de referencia teórico sobre el que posteriormente se desarrollará la modificación de conducta. Poco después aparecerán las primeras formulaciones de Watson sobre el conductismo donde el objeto de la psicología es la conducta y la forma de estudiarla sigue los pasos desarrollados por Pavlov y Thorndike.

En 1913 Watson proclama un manifiesto sobre una nueva clase de psicología: El conductismo. Rechazó que existiera alguna diferencia entre el estructuralismo y el funcionalismo ya que ambos habían adoptado la definición tradicional de la psicología como "la ciencia de los fenómenos de conciencia". Watson se distanciaba de la psicología tradicional al estar limitado por el mentalismo, no podía trabajar con animales ya que no eran sujetos "con contenidos conscientes". Por lo tanto ahora proponía utilizar seres humanos como sujetos y emplear métodos de investigación que fueran exactamente comparables a los que se usaban en el trabajo con animales. La cuestión principal para Watson era que no existían procesos mentales funcionales que representen un papel causal en la determinación de la conducta. Solo existían cadenas de conductas, algunas de las cuales eran difíciles de observar. Afirmaba que el pensamiento no implicaba al cerebro. Watson defendía que no hay procesos iniciados centralmente, siempre que hay pensamiento hay débiles contracciones de los sistemas musculares implicados en el ejercicio abierto del acto. Watson utilizó los métodos pavlovianos aplicados a sujetos humanos se convertirían en la herramienta de investigación del conductista, y la teoría de los reflejos condicionados seria la base para la predicción y el control de la conducta de animales y personas (Leahey, 1998).

Unos años más tarde del fin de la guerra aparecen las grandes teorías neoconductistas del aprendizaje de Hull, Mowrer y Tolman, en lo que sería la "Edad de Oro del Conductismo", sobresaliendo especialmente el modelo de trabajo de Skinner que supone el desarrollo de leyes específicas del comportamiento sobre las cuales se asentarán las directrices de la intervención. Publica trabajos sobre el condicionamiento operante y desarrollos consecuentes como $\mathrm{La}$ 
conducta de los organismos (1938) y Ciencia y conducta humana (1953). Estas publicaciones empiezan a introducir nuevos términos y aportan claras directrices sobre cómo aplicar los principios del aprendizaje para la modificación y mejora del comportamiento humano y la solución de trastornos de conducta.

De acuerdo con Skinner la conducta debe ser explicable, predecible y modificable atendiendo a las relaciones funcionales con sus antecedentes y consecuentes ambientales y rechazando las explicaciones basadas en constructos internos inferidos. En estos momentos y tras la segunda guerra mundial, aparece una gran demanda de profesionales que puedan intervenir con éxito a las personas que han sido afectadas. La modificación de conducta aparece como una alternativa innovadora, válida y eficaz, fundamentada en un cuerpo teórico solido capaz de explicar los trastornos del comportamiento y presentar soluciones a estos. Hacia finales de los años 50 ya pueden señalarse tres focos principales de movimientos científicos conductuales, EEUU (con Skinner \& Lindsley), Sudáfrica (Wolpe) e Inglaterra (Eysenck).

En la década de los sesenta las técnicas de modificación de conducta empiezan a expandirse rápidamente, aplicándose con gran éxito a problemas que hasta ese momento carecían de tratamiento. Se aplican técnicas operantes a la implantación del lenguaje, retraso mental, autismo y delincuencia así como a trastornos de conducta en niños normales. Se desarrollan las técnicas de economía de fichas, se analizan y crean nuevas variables para la sensibilización sistemática. Se desarrollan técnicas de habilidades sociales, inundación y prevención de respuesta. Durante este periodo llamado de consolidación de la terapia de conducta las características principales son:

1. Se enfatiza las conductas y sucesos observables del individuo, tratándolas directamente, sin prestar atención a los supuestos procesos patológicos subyacentes.

2. Se describen los trastornos de conducta como una relación estimulo, respuesta y consecuencia (E-R-C). El análisis funcional es la base para la explicación de los problemas de conducta.

3. Se individualiza cada tratamiento, qué tratamiento para qué tipo de cliente con qué problemas y su costo (Paul, 1969).

4. Las conclusiones extraídas en el laboratorio se aplican y desarrollan en la vida cotidiana. Mucha aplicación y poca teoría.

5. Los programas de tratamiento son simples, manejándose pocas variables con tratamientos con reducidos componentes. 
Aparece una disociación entre la investigación de psicología básica y la aplicación de la modificación de conducta. La psicología experimental comienza a dejar de lado el aprendizaje y los modelos de condicionamiento para centrarse en procesos cognitivos (memoria, percepción, atención) mientras que la modificación de conducta están inmersos en la aplicación de sus trabajos sin prestar atención en la investigación básica.

\section{Segunda Generación: La era Cognitivista (1960)}

La psicología cognitiva surge como corriente psicológica entre los años 50 y 60 de una manera muy débil y como reacción al conductismo. Autores como Jean Piaget y sus trabajos (ignorado por EE.UU) comenzaron a leerse en la época de los 60 cuando muchos psicólogos nobeles desencantados con el conductismo comienzan a investigar sobre el desarrollo cognitivo. Sin embargo hubo una teoría sobre la disonancia cognitiva muy influyente en la década de los 50 de Leon Festinger sobre las creencias de las personas y sus interacciones (Cognición-Conducta).

El término comenzó a usarse con la publicación del libro Cognitive Psychology por Ulrich Neisser en 1967. Pero la aproximación cognitiva había sido traída a un primer plano tras la publicación del libro de Donald Broadbent Percepción y Comunicación en 1958. Desde ese momento, la metáfora dominante en el área ha sido el modelo de procesamiento de información de Broadbent.

Los años setenta están marcados por la incorporación de variables cognitivas en la explicación de los problemas comportamentales y en el tratamiento. Se distinguen claramente dos enfoques: (Pelechano, 1979)

- Continuistas: Los que consideran que estos eventos internos están determinados por las mismas leyes E-R que las conductas manifiestas, como Cautela. Aplican los modelos de condicionamiento en la explicación y modificación de las cogniciones.

- Rupturistas: Los que consideran que las cogniciones tienen un rol causal en la conducta y no se explican exclusivamente por procesos de condicionamiento. Incluyen modelos y técnicas cognitivas específicas. Autores como Beck, D'Zurilla, Goldfried, Meichenbaum o Mahoney.

Se enfatiza en las intervenciones el entrenamiento al cliente de habilidades de autocontrol, para aumentar la generalización de las ganancias, así como dotarles de recursos para hacer frente a las situaciones problemáticas nuevas. Se encuentran novedades como el uso de la exposición en vivo y la aplicación del entrenamiento en habilidades sociales más complejas.

La psicología cognitiva hace uso de procesos mentales para explicar la conducta (a diferencia de tan solo asociaciones entre estímulos y respuestas). Los psicólogos cognitivos ponen énfasis en la influencia que el procesamiento 
de la información tiene sobre la conducta, afirmando que el individuo compara la información nueva con su "esquema" o estructura cognitiva preexistente. Los acontecimientos y las situaciones nuevas se interpretan a la luz de lo que ya se ha aprendido. Entre las características comunes de la Orientación CognitivoConductual se encuentran:

1. El cambio conductual está mediado por las actividades cognitivas. La identificación y alteración de aspectos cognitivos producirá el cambio deseado.

2. Determinismo reciproco entre pensamiento, ambiente y conducta.

3. La terapia está diseñada para ayudar al paciente a identificar, probar la realidad y corregir las creencias y pensamientos disfuncionales.

4. Técnicas aplicadas: Restructuración cognitiva, solución de problemas, entrenamiento autoinstruccional...

5. Relación terapéutica colaborativa enfatizando el rol activo del paciente.

\section{Tercera Generación: Las Terapias Contextuales (1984)}

En 1984 Steven C. Hayes publica el primer artículo (Makingsense of Spirituality) sobre una nueva generación de terapia y en 1987 el primer capítulo (A contextual approach to therapeutic change) de un libro llamado Psychotherapists in clinical practice: Cognitive and behavioral perspectives, donde se recogían el tratamiento y extensión de diferentes casos clínicos. Es a partir de estos años donde se puede considerar el germen de las terapias contextuales comienza a desarrollarse.

La primera generación de técnicas conductuales estaba fundamentada directamente sobre el condicionamiento clásico y el operante. Esta primera generación de terapias tiene un carácter empírico, experimental y se centra en el cambio directo de la conducta. Surgen, por un lado, ante las dificultades de las terapias de modificación de conducta más tradicionales para poder modificar comportamientos en la vida diaria de la persona, sin control directo de contingencias, y para poder actuar sobre los problemas a partir de una hora de sesión clínica con individuos adultos. La segunda generación, incorporó las técnicas cognitivas. Ante las dificultades para abordar inicialmente los temas más cognitivos, pensamientos, obsesiones, recuerdos, traumas, etc., con las técnicas de modificación de conducta, que habían sido suplidas en su explicación por las denominadas cognitivas-conductuales. Aquí se comienza a cambiar pensamientos y recuerdos como cualquier otra conducta, a partir del lenguaje, pero sin misterios ni teorías profundas sobre la mente del individuo. Actualmente, se ha abierto una tercera generación, en la que, sin perder de vista la base experimental y científica, se propone una recuperación del planteamiento contextualista, se renueva el énfasis en el análisis funcional y se profundiza en el papel que la relación terapéutica y 
el lenguaje natural —el que acontece en la consulta — juegan en la clínica. Estas terapias de tercera generación adoptan una perspectiva más experiencial y opta por estrategias de cambio de carácter indirecto. Se da relevancia al contexto y no al modo en que se presentan las conductas.

Las diferencias de estas terapias con las de segunda generación son que estas enfocan los casos desde una perspectiva contextualista, individual, cuyo principal objetivo no es la reducción de síntomas que presenta el paciente sino una reorientación de la vida de la persona, sin importar si los síntomas se reducen o no. Mientras que las cognitivas tienen un enfoque de déficit o defecto de la persona, presupone que la causa del trastorno es un defecto/déficit en el funcionamiento psicológico de la persona y el objetivo principal es la reducción de los síntomas que presenta el paciente, que definirá la eficacia de la terapia (a mayor reducción de síntomas, más eficaz es la terapia).

Por lo tanto las terapias de "tercera ola de conducta" explican los problemas psicológicos en términos interactivos, funcionales y contextuales, algo que se da en la evolución de la persona y sus circunstancias, no en términos de déficit o defecto de la persona. No se entiende que el problema o trastorno se encuentre dentro de la persona, sino que es la persona la que se encuentra en unas circunstancias, una situación problemática.

\section{TERAPIAS CONTEXTUALES FUNCIONALES DE GRUPO: FUNDAMENTOS, EVIDENCIAS, EFICIENCIA Y APLICACIONES}

Los dos principales fundamentos teóricos y empíricos de las Terapias Contextuales y Funcionales de Grupo están en la Teoría del Marco Relacional (Barnes-Holmes y cols., 2001; Torneke, 2016 y Villate, 2016) y el Socioconductismo (Guerin, 2015, 2016, 2017); y así lo solemos argumentar desde la doble perspectiva filosóficaexperimental y sociopsicológica (Ruiz, 2017).

La Teoría del Marco Relacional (TMR/RFT) supone un cuerpo de investigación basado en como a través del lenguaje los humanos establecen relaciones arbitrarias entre los eventos de su vida como una operante relacional generalizada, y como esto afecta a las vidas de las personas de manera poderosa tanto para bien como para mal.

El Socioconductismo destaca el papel de las prácticas sociales en la conformación de la conducta y aquello que consideramos normal y anormal mediante la interacción continua de los seres humanos en instituciones de poder y control.

En el campo de la psicología clínica y la psicoterapia, y aún en la terapia o modificación de conducta, la mayor parte de la investigación y proliferación de manuales y guías de terapia se ha venido centrando en los formatos de terapia 
individual. Esto parece obedecer al "interés de lo individual" desde una concepción ideológica neoliberal de manifiesto tanto en las políticas e intereses universitarios occidentales, como en los mismos terapeutas de referencia mundial y sus grupos de investigación muchas veces un tanto alejados de las políticas públicas sanitarias. Esta concepción de la psicología y de la misma psicoterapia parece atravesar todas las corrientes dominantes de la psicología con honrosas excepciones entre modelos tan diferentes como el psicodinámico (por ejemplo la concepción de los Grupos Operativos de Pichón Riviere, 1987, 1995, 1999) como desde el conductismo radical (como el caso del Socioconductismo de Bernard Guerin, 2015, 2016, 2017). Habitualmente, aunque no siempre es el caso, las concepciones más sociales o grupales parecen nutrirse de ideologías más progresistas, con lo que al menos, en psicología, la relación entre ideología y ciencia parece evidente. La Terapia de Grupo suele ser un referente en lo público, aunque eso sí, habitualmente infrautilizada a pesar de su soporte empírico, siendo el modelo de lo individual aún el imperante entre los clínicos de los servicios públicos.

El mayor contacto entre los investigadores y clínicos públicos y privados, y entre la universidad y las instituciones públicas sanitarias puede fomentar un intercambio fructífero donde las ideologías y las prácticas sean más convergentes y más flexibles en ambas direcciones.

En general, las distintas psicoterapias que se han mostrados efectivas con soporte empírico, lo son además en formato grupal. Además de la efectividad o resultados adecuados que suelen ser similar en ambos formatos, la terapia grupal tiene una ventaja importante sobre la individual, que es su eficiencia: Podemos ayudar a más personas en un tiempo determinado; siendo esto clave en los servicios públicos de salud mental a menudo masificados ante la gran demanda de personas que piden ayuda psicológica.

Un trabajo ya clásico de referencia sobre las terapias basadas en la evidencia fue realizado por Smith, Glass \& Miller (1980) que comparó la eficacia de diferentes modelos de psicoterapia (estando basado el $49 \%$ de ellas en terapias de grupo) con el resultado de que eran efectivas y además las diferencias entre las distintas corrientes teóricas eran mínimas; demostrando adicionalmente que las terapias grupales eran igual de efectivas que las individuales. Otros estudios posteriores redundan en resultados similares como veremos a continuación en la familias de terapias cognitivas-conductuales tanto en su líneas más hibridas como radicales.

Sobre la teorización de los factores que hacen eficaz a una terapia de grupo, de manera independiente a su propio marco teórico, la mayoría de los autores suelen tomar como referencia los 11factores comunes listados por Yalom (1985) y Vinogradov y Yalom (1996) : la universalidad (los miembros del grupo se tranquilizan cuando perciben que sus compañeros de grupo tienen problemas 
similares a los suyos; el altruismo (dar ayuda a otros eleva la satisfacción personal, además de ser útil para quien la recibe); cohesión grupal (la sensación de pertenencia a un grupo suele valorarse especialmente para quienes previamente se han sentido solo); socialización (el grupo puede ofrecer oportunidades para aprender patrones de relación social más adecuados); imitación o modelado (cuando el grupo funciona bien los miembros del grupo suelen adoptar comportamientos adaptativos de otros); aprendizaje interpersonal (la propia relación entre los miembros del grupo puede modificar las conductas sociales de relación por otras más adaptativas); recapitulación del grupo familiar primario ( muchas de las relaciones de los miembros del grupo se basan en aprendizajes derivados de sus relaciones familiares que se pueden modificar en la experiencia grupal); infundir esperanza (observar la mejoría de otros miembros del grupo refuerza seguir en la terapia); información participativa (los consejos y el intercambio de experiencias forman parte del proceso grupal); catarsis (compartir emociones evitadas que son aceptadas por el grupo es una experiencia importante y valiosa en los grupos) y enfrentarse a factores existenciales (en los grupos temas relevantes como la responsabilidad, el enfrentamiento al miedo a la muerte y el tema de los valores pueden ser claves en la experiencia grupal).

De manera más reciente desde una perspectiva de elementos efectivos de la relación terapéutica Norcross \& Wamplond (2011) han estudiado que elementos de la relación terapéutica terapia de grupo son los más efectivos, destacando los siguientes: la alianza terapéutica (acuerdos sobre los objetivos, tareas y tipo de relación); la cohesión grupal, la empatía y recoger el feedback que dan los miembros del grupo a las intervenciones del terapeuta y compañeros del grupo.

La terapia de grupo dada su eficacia y eficiencia debería formar parte de la cartera de servicios psicológicos de cualquier la red de salud mental pública que se aprecie (Fernández Liria, Rodríguez Vega, Muñoz y Cebolla, 2012).

El movimiento de terapias de grupo con soporte empírico, aunque menos estudiado que los formatos individuales, es un campo de investigación en alza, e incluso cuenta con una organización específica dedicada a esto: A.G.P.A (American Group Psychotherapy Association. http://www.agpa.org/home).

Entre los distintos modelos de terapia grupal con soporte empírico suele destacar las de enfoque cognitivo-conductual por su mayor número de comprobaciones de eficacia y eficiencia. En este punto se engloban tanto las terapias grupales de primera, segunda y tercera generación cognitiva-conductual.

Las de segunda generación han sido las más estudiadas hasta el momento en cuanto a su soporte empírico en multitud de problemas diagnosticados desde las categorías psiquiátricas vigentes (CIE, DSM). Estos enfoques "híbridos" suelen mezclar teorías cognitivas de déficit o vulnerabilidad en forma de disposiciones 
internas de tipo neurobiológico o temperamental, sistema de creencias o esquemas disfuncionales y déficits de habilidades; junto a formas de aprendizaje respondiente, operante y vicario. En estas terapias grupales de segunda generación el foco de trabajo consiste habitualmente en aprender habilidades para que los sujetos reduzcan o modifiquen su malestar emocional por conductas más sanas o saludables. El hecho es que se han mostrado eficientes y eficaces, amén de que se les pueda criticar los problemas que conllevan su alianza o cercanía con el modelo psiquiátrico de enfermedad y la no demostración de que el uso que hacen de sus componentes cognitivos-verbales sea más eficaz que sus componentes más específicamente conductuales, por ejemplo la reestructuración cognitiva frente a la activación conductual; siendo el caso habitual justo el contrario (Ruiz, Ruiz y Andújar, 2016).

Sea como sea, el hecho es que el modelo híbrido de segunda generación se ha mostrado eficaz y eficiente en numerosas problemáticas; así como las terapias contextuales funcionales de tercera generación, algunas de ellas aún en curso de validación en formato grupal (como el caso de la Psicoterapia Analítica Funcional de Grupo). Presentamos un resumen de algunos de estos trabajos con soporte empírico en la tabla 1.

\section{Los focos de trabajo de la ACT de Grupo}

En la ACT de grupo es esencial que los participantes se den cuenta y sean conscientes (discriminen) tres aspectos de su funcionamiento psicológico: las diferencias entre los contenidos mentales (experiencia mental interna) y los procesos de cómo les va en su vida con los efectos contingenciales de su conducta (experiencia de los 5 sentidos); las diferencias entre luchar y quedarse enganchados (alejarse) en los intentos infructuosos para eliminar las experiencia mentales y emocionales no deseadas a corto plazo (evitación experiencial) de acercarse activándose hacia acciones valiosas en función de los valores personales a largo plazo; y como consecuencia de todo lo anterior generar un marco deíctico desde el yo observador donde el sujeto no se identifique o fusione con ninguno de sus contenidos mentales ni sus conductas específicas para generar flexibilidad psicológica.

En esto seguimos fundamentalmente las propuesta de Kevin Polk (Polk, 2009 y Polk \& Schoendorff, 2014, 2016) usando con frecuencia la Matrix, el bolígrafo y otros elementos visuales y experienciales, como la representación dramatizada de las metáforas que no solo son verbalizadas y otros ejercicios experienciales procurando que todo el grupo participe en ellos, recabando las respuestas de los sujetos a estos y moldeándolas en relación a los tres focos referidos anteriormente. En la misma línea usamos los ejercicios de mindfulness tanto en vivo en las sesiones del grupo como grabados para su práctica entre sesiones, usando reiteradamente en el grupo el juego de 4 cuestiones básicas, que nos sirve para recabar feedback 
de las conductas clínicamente relevantes y su moldeamiento gradual hacia las discriminaciones más adecuadas:

-¿Esta semana o ahora aquí mismo en el grupo, has luchado por no sentir o pensar cosas que te desagradan y sin embargo las sigues pensando y sintiendo? (esto se pone en relación al moldeamiento la "desesperanza creadora" de las luchas y evitaciones infructuosas, paralizadoras o alejamientos de la sendas valiosas).

- ¿Esta semana has cultivado las rosas de tu jardín aunque tenías alguna piedra-garbanzo en tu zapato? (Previamente hemos implicado al grupo a meterse un garbanzo en sus zapatos y preguntado qué harían para moverse en la habitación en una dirección valiosa-señalando una dirección de la sala, habiéndose dado cuenta de que no pueden sacarse ese garbanzo muchas veces, que pasa si se dedican a parar y tratar de sacarlo a toda costa, etc.).

-¿Esta semana o aquí en el grupo, en algún momento has notado la diferencia entre la experiencia de los 5 sentidos y la de juzgar con la mente? (aquí los ejercicios y metáforas de defusión son usados para moldear esta discriminación). En este aspecto es fundamental trabajar el darse cuenta o discriminar las diferencias entre los deícticos yo allí y entonces (enganchesfusiones en el pasado/futuro) del yo aquí y ahora (experiencias sensoriales del entorno y posibilidades de acción presentes)

- ¿Esta semana o aquí y ahora crees que tus problemas psicológicos son una enfermedad? (aquí usamos la dramatización de los efectos sobre el mayor o menor empoderamiento de mantener que los problemas provienen del interior de la cabeza y que deben ser "desentrañados por un experto ajenos a ellos mismos frente a que los problemas derivan de sus circunstancias de vida y lo que hacen en ellas. Evitamos discutir las teorías de los miembros del grupo y planteamos en esto los efectos funcionales de sus creencias sobre los problemas psicológicos).

Con todas estas experiencias grupales planteadas en estos tres focos, más el adicional del modelo personal de problema psicológico enfermedad versus problemas de la vida que basamos en los planteamientos de Bernard Guerin (Guerin, 2015, 2016, 2017) buscamos aumentar tanto la flexibilidad psicológica (defusión de contenidos verbales que hacen que las personas reiteren acciones infructuosas corto-plazo y activación en acciones valiosas a largo plazo) como el empoderamiento personal. Se busca pues "derivar" todas estas respuestas de acciones valiosas a largo plazo junto al darse cuenta (CCR2). 


\section{Tabla 1}

Eficacia y Eficiencia de las Terapias de Grupo Cognitivas-Conductuales. Algunos estudios

\begin{tabular}{|c|c|c|}
\hline Área problemática & Segunda Generación & Tercera Generación \\
\hline $\begin{array}{l}\text { 1. Ansiedad-Depresión } \\
\text { en general. Distímia y } \\
\text { Depresión Mayor en } \\
\text { Adultos }\end{array}$ & $\begin{array}{l}\text {-Meta análisis de Burlingame, } \\
\text { Strauss \& Joyce, } 2013\end{array}$ & $\begin{array}{l}\text {-Chu y cols.2009 } \\
\text {-Jhonson Norton, } 2010 \\
\text {-Soleimani y cols. } 2015 \\
\text {-Fereidoini y cols. } 2015\end{array}$ \\
\hline $\begin{array}{l}\text { 2. Ansiedad-Depresión } \\
\text { y otros problemas } \\
\text { emocionales en general en } \\
\text { niños y adolescentes }\end{array}$ & $\begin{array}{l}\text {-Meta análisis de Power, } \\
\text { Sigmmarson \& Emmelkamp, } \\
2008\end{array}$ & $\begin{array}{l}\text {-Swain, Hancock, Dixon, Koo y Bowman, } \\
2013 \\
\text {-Gómez, Luciano, Páez-Blarrina, Valdivia- } \\
\text { Salas y Gil-Luciano, } 2014 \text { (impulsividad en } \\
\text { adolescentes) } \\
\text {-Padilla Torres y Jiménez Arriero, } 2014\end{array}$ \\
\hline 3.Ansiedad Social & $\begin{array}{l}\text {-Meta análisis de Power, } \\
\text { Sigmmarson \& Emmelkamp, } \\
2008\end{array}$ & $\begin{array}{l}\text {-Myler, } 2012 \\
\text {-Boone \& Manning, } 2012 \\
\text {-Monajeni y Habibi, } 2015 \\
\text {-Fleming \& Kocovsky, } 2014\end{array}$ \\
\hline 4.Trastorno de Pánico & $\begin{array}{l}\text {-Austin y cols. } 2008 \\
\text {-Bohni y cols. } 2009\end{array}$ & $\begin{array}{l}\text {-Quismondo, Rios, Pedraza, Liria y Lahea, } \\
2016\end{array}$ \\
\hline $\begin{array}{l}\text { 5. Trastorno por Estrés } \\
\text { Postraumático }\end{array}$ & $\begin{array}{l}\text {-Alvarez, McLean, } \\
\text { Harris, Rosen, Ruzek y } \\
\text { Kimerling,2011 }\end{array}$ & -Treloar, 2011 \\
\hline $\begin{array}{l}\text { 6. Trastorno Obsesivo- } \\
\text { Compulsivo }\end{array}$ & $\begin{array}{l}\text {-Meta análisis de Jonsson \& } \\
\text { Hougaard, } 2008\end{array}$ & $\begin{array}{l}\text {-Existe un centro en los Ángeles (EEUU) } \\
\text { dedicado al tratamiento de estos problemas } \\
\text { en terapia de grupo: ¿Evidencia/Eficiencia? } \\
\text { http://ocdla.com/ocdtherapygroups }\end{array}$ \\
\hline $\begin{array}{l}\text { 7. Problemas de } \\
\text { ansiedad relacionados } \\
\text { con somatizaciones e } \\
\text { Hipocondría }\end{array}$ & $\begin{array}{l}\text {-García-Campayo, Pascual, } \\
\text { Alda y Oliván, } 2005\end{array}$ & -Eilenberg, Frostholm \& Kronstrand, 2014 \\
\hline $\begin{array}{l}\text { 8.Bulimia y Trastornos } \\
\text { Alimentarios }\end{array}$ & -Burlingame y cols. 2013 & Juarascio y cols. 2013 \\
\hline $\begin{array}{l}\text { 9.Estrés y malestar } \\
\text { emocional asociado a } \\
\text { enfermedades médicas } \\
\text { incapacitantes y Cancer }\end{array}$ & -Applebaum y cols. 2012 & $\begin{array}{l}\text {-Hadlansmyth, White, Nesin \& Greco, } \\
2013 \text { (adolescentes con diabetes) } \\
\text {-Wittingham, Sanders, Mckinley \& Boyd, } \\
2014 \text { (orientación a padres con hijos con } \\
\text { daño cerebral) } \\
\text {-Soto, Barrechenea-Arando y Estruch, } \\
2013 \text { (pacientes con fibromialgia) } \\
\text {-Hopko y cols. } 2014 \text { (pacientes oncológicos } \\
\text { con cáncer) }\end{array}$ \\
\hline $\begin{array}{l}\text { 10.Trastornos de la } \\
\text { Personalidad }\end{array}$ & $\begin{array}{l}\text {-Blum y cols. } 2002 \text { y } 2008 \\
\text {-Boss y cols. } 2010 \\
\text {-Harvey y cols, } 2010\end{array}$ & $\begin{array}{l}\text {-Linehan y col., } 2006 \\
\text {-Soler y cols. } 2009 \\
\text {-Urbano y Love, } 2011 \\
\text {-Guteling y cols. } 2012 \\
\text {-Clarke, Kingston, James, Bolderston \& } \\
\text { Remington, } 2013 \\
\text {-Hoekstra, 2008 (FAP) } \\
\text {-Vanderbeghe, 2015 (FAP) }\end{array}$ \\
\hline $\begin{array}{l}\text { 11.Afrontamiento del Estrés } \\
\text { y la Ira }\end{array}$ & -DiGiuseppe y Tafrate, 2003 & -Daltry (2015) \\
\hline $\begin{array}{l}\text { 12.Trastornos del Consumo } \\
\text { de Sustancias }\end{array}$ & $\begin{array}{l}\text {-Hervás, Gradoli, Gurrea, } \\
\text { Bou y Gallus, } 2002\end{array}$ & $\begin{array}{l}\text {-Dimeff, y Linehan, } 2008 \\
\text {-Agevli y cols. } 2007\end{array}$ \\
\hline
\end{tabular}


13. Psicosis y Esquizofrenia

Kanas, 1986,1996

-Burlingame, McKenzie \&

Strauss, 2004

-Burlingame, Strauss\&y

Joyce, 2013
-Meta análisis de Tonarelli y cols. 2016

-Castilho, Pinto, Viega, Carvalho y

Madeira, 2015

\section{EI papel de la metáfora y los ejercicios experienciales en la ACT de Grupo}

Es inevitable el uso metafórico en el lenguaje, así que de una u otra forma probablemente (si es usted terapeuta) esté utilizando metáforas con sus clientes. La comunidad verbal usa metáforas así como historias, mitos, alegorías y cuentos; recursos narrativos que la humanidad ha utilizado para transmitir de generación en generación las experiencias y la percepción de la realidad y/o hechos que acontecían en cada cultura. Por esta razón la metáfora es muy útil en psicoterapia, porque se ha estado utilizando durante la historia para comprender rápidamente el conocimiento simbólico en áreas más complejas. Para Foody (2014) su propósito común en la configuración clínica es doble. En primer lugar, están diseñados para validar la experiencia del cliente. En segundo lugar, tienen como objetivo mejorar la conciencia del cliente sobre su situación.

Para conseguir el efecto terapéutico más potente de la metáfora es importante tratar de entender y comprender los diferentes contextos en los que la persona se mueve e interactúa. Las metáforas pueden influir en el modo en el que se piensa y por lo tanto en cómo se actúa, dando así diferentes soluciones ante una misma situación. En una seria de cinco experimentos Thibodeau \& Boroditsky (2011) demostraron cómo hablar de un tema complejo de una manera metafórica u otra puede llevar a diferentes acciones para solucionarlo.

Utilizar metáforas en las intervenciones psicológicas puede ayudar a crear realidades, sobre todo sociales. De este modo, la utilización de la metáfora en terapia grupal ayuda a crear en cada uno de los participantes una realidad y una especie de guía para la acción ajustando paralelamente la metáfora a la vida cotidiana. En su libro, Lakoff y Johnson, Metáforas de la vida cotidiana (1980) escriben cómo las metáforas pueden servir como "profecías que se cumplen" para las personas ya que pueden agitar al oyente, darse cuenta y orientarse hacia el cambio. En este sentido la metáfora es un elemento clave en la terapia a la hora de ayudar a entender al usuario del grupo cómo funcionan las cosas, con el fin de actuar en diferentes situaciones que sean similares funcionalmente. La metáfora actúa como un traje a medida donde la propia persona que lo lleva elige, con su experiencia, cómo (con qué medidas) ajustarlo a su cuerpo.

Nuestra propia experiencia en la utilización de metáforas en Grupos nos ha demostrado que son útiles para ayudar al paciente a discriminar entre dos aspectos básicos bajo nuestro punto de vista en la intervención grupal (Ver tabla 2). Por un lado discriminar los contenidos y los procesos, es decir diferenciar entre el contenido mental (fusión cognitiva) y el experiencial (o de los 5 sentidos). Y por 
otro lado ayudar a la persona a diferenciar entre aproximaciones a valores (acciones específicas orientadas hacia lo que realmente le importa) frente a evitaciones (intentos de control de los eventos privados que por lo general son infructuosos y general más contenido desagradable). Al mismo tiempo que se acompaña de ejercicios de relajación y meditación para trabajar el "Yo observador" (darse cuenta de sensaciones). La metáfora que vertebra esta forma de trabajo durante todas las sesiones grupales es la metáfora del Jardín de la vida adaptado por Ruiz y Trillo (2017) en la que se le cuenta a los participantes del grupo que imaginen poseen un jardín donde pueden plantar sus rosas. A partir de ahí se identifican rosas como valores y acciones concretas para cuidar dichas rosas con el fin de mantenerlas bonitas y agradables. Ser consciente de que prestar más atención a las malas hierbas haría que pasáramos menos tiempo cuidando nuestras rosas ayuda al participante del grupo a reconocer qué hace que no le funciona y que acciones si al vivir su vida.

\section{Nuestra experiencia con la ACT de Grupo con problemas ansiosos- depresivos}

En nuestro servicio ambulatorio de salud mental comunitaria las personas son derivadas desde los dispositivos de atención primaria (ambulatorios) por el médico de cabecera. La agenda de los psicólogos de este servicio suele rondar unos 9 pacientes diarios citados a razón de media hora por consulta. De esas 9 citas dos de ellas suelen ser pacientes derivados por primera vez, y las 7 restantes revisiones. El anterior contexto de trabajo genera, entre otras dificultades, que al cabo de cierto tiempo que se produzcan largas listas de espera para las citas individuales (a veces de 3 semanas o más entre sesiones). Esto suele producir masificación y malestar tanto en los pacientes (clientes o usuarios según concepto no menor) como en los clínicos del servicio. A pesar de ello la mayoría sigue trabajando con estas agendas de citas individuales.

Tabla 2

Eficacia y Eficiencia de las Terapias de Grupo Cognitivas-Conductuales.

\begin{tabular}{ll}
\hline Discriminaciones & Metáforas \\
\hline 1. Contenidos y Procesos & Piedra en el zapato \\
2. Aproximaciones a Valores y Evitación & Piloto automático \\
& El bolígrafo \\
& El jardín de la vida \\
3. Yo Observador & El conductor del autobús \\
& Espectador en el cine \\
& Las troncos en el río \\
\hline
\end{tabular}

Nosotros venimos trabajando con grupo como alternativa, imperfecta y limitada sin duda, a la anterior situación; pero con mayores oportunidades de cambios respecto a la modalidad anterior, según nuestros datos recabados y experiencia, que llega a rondar el $80 \%$ de cambios positivos en la modalidad grupal de sus integrantes frente a un $50 \%$ de cambios en la modalidad individual 
con "diagnósticos" similares. Además la tasa de abandonos con la modalidad de citas individuales es mayor que en la grupal en este contexto. Nuestra conducta como trabajadores con grupos es reforzada en gran parte por estos resultados.

Aquí presentamos la experiencia resumida con uno de estos grupos en la última sesión, donde acuden 10 de sus integrantes (en el inicio llegaron a participar 13 personas).

Habitualmente en estas sesiones solo está un terapeuta, y ocasionalmente acompañado por un psicólogo en formación. En esta ocasión contamos con otro psicólogo, además del habitual, con experiencia en ACT y FAP.

Comenzamos el grupo dando la bienvenida a todos y agradeciendo el trabajo que han venido realizando a lo largo de las sesiones y recordándoles que nos encontramos al final de la terapia y veremos que han recabado en su jardín con el cultivo de las rosas y si han cambiado su lucha con las malas hierbas.

Se continua la sesión con la realización de la hoja de evolución semanal con las "rosas" y las "malas hierbas" (Cuestionario de Jardín de tu Vida de Trillo y Ruiz, y AAQ-II reducido de 7 ítems). Al ser la última sesión también se les pide rellenar el Cuestionario de Valoración final de la experiencia grupal.

Después de rellenar los cuestionarios se les plantea "la metáfora del aguilucho herido" para evocar la experiencia vivida en el grupo con el hecho de tener que volar ahora solo/a sin el grupo al llegar a su finalización, o sea seguir adelante sin vernos una vez en semana. Con ello pretendemos explorar temas de dependencia grupal, autonomía alcanzada en formas de rosas y malas hierbas, en relación a derivaciones de acciones valiosas más allá de venir, apoyarse y desahogarse en el grupo; de tomar notas de sus conductas clínicamente relevantes.

Básicamente esta metáfora habla de que un granjero se encuentra en el campo un aguilucho herido, le cura y le protege en su granja poniéndolo con pollitos a comer y a hacer su vida con ellos, llevando esta situación a que el aguilucho se comporte como un pollito, picotee como un pollito, de saltitos como un pollito, etc. En esto que un día un ecologista pasea por el campo y ve el aguilucho en la granja y le pregunta por ello al granjero, que le cuenta lo sucedido. De hecho habla con este del gran trabajo de cuidado realizado, pero que el aguilucho tiene más potencial que tenerlo aquí como un pollito más. El granjero no está muy convencido pero accede a que el ecologista le dé la oportunidad de emprender el vuelo por los cielos, cosa que se produce tras varios intentos infructuosos.

A continuación se les pregunta si se ven como pollitos o más cerca de desplegar sus alas hacia sus rosas. Es frecuente en nuestra experiencia que en varias personas de los grupos se produzca cierta dificultad para dejar este soporte semanal, y más en este contexto de citas individuales cortas y espaciadas. En la 


\section{tabla 2 se resumen las conductas clínicamente relevantes la experiencia de uno de estos grupos en su sesión final.}

Tabla 3

Metáforas utilizadas en intervención grupal

\begin{tabular}{ll}
\hline $\begin{array}{l}\text { Nombre y barrera de malas } \\
\text { hierbas }\end{array}$ & $\begin{array}{l}\text { Conducta clínicamente } \\
\text { relevante-2 II. "Darse cuenta" } \\
\text { (discriminar) }\end{array}$
\end{tabular}

1- Diego (miedo a coger el coche) "Me he dado cuenta de que una vez que cojo el coche y me vuelvo no me sirve"

2- Gregorio (desacuerdos con su madre, desánimo y paro laboral)

3- José (Diagnosticado de Tr.Bipolar-No tener sentimientos y estar en stanby)

4- Paco (Conflicto con familia y exceso de trabajo)

\section{5- Noemi (Diagnosticada de Trastorno Mental Grave) \\ 6- Simón (Ruptura sentimental)}

\section{7- Manuela (Duelo por fallecimiento del marido)}

8- Juana (Enfermedad orgánica en revisión y/o diagnóstico de tno. conversivo)

9- Ramona (Muy ansiosa al volver a su trabajo habitual, frecuentes suspiros sonoros en las sesiones)

10- Rosa (Obsesiones)

\section{"Me preguntaba si esto es la realidad o mi mente. Ahora veo que es la realidad pero no yo"}

"Me doy cuenta de que soy un holgazán, me tengo que activar"

"Se lo hacía pasar mal a mi familia, bebía mucha Coca-Cola y creaba conflicto"

"El grupo me ha servido para estar mejor. El haber conocido al grupo me ha gustado"

\section{"La ruptura ha sido dolorosa pero me ha ayudado a frenar mi excesivo ritmo de trabajo"}

"He salido del pozo aunque hay
días que lloro y rabio y luego
pienso, ¿de qué sirve esto?"
"Tengo las alas cerradas, creía que
podía cambiar las cosas y no me
está funcionando nada. Cada vez
estoy peor
"No me ha ido bien, sigo llorando.
Me cierro más porque no se
expresarme"
"Me ha ido bien en general. Estoy
más contenta"

\author{
Conducta clínicamente \\ relevante-3. "Acciones nuevas \\ llevadas o previstas hacia las \\ rosas"
}
"Sigo adelante y conduzco porque me gusta. Pienso que es bonito viajar. Viajo con mis miedos. Además cultivo a mi familia y bien"
"Sigo buscando trabajo y pongo carteles para promocionar lo que ofrezco de trabajo, para la aceituna, etc. A lo mejor tengo que darle cariño a mi madre y no rencor"

"Me quiero activar pero preocuparme no sirve para esto"

"Ahora tengo cariño, me río en casa, me valgo por mí mismo. Atiendo más a mi familia y trabajo menos en la tienda"

"Me he apuntado a un grupo de teatro y he conocido a gente nueva, quería tener vida social"

"Dedico los fines de semana a salir con los amigos, y dejo el trabajo de lado los fines de semana"

"Voy a andar, a clases de yoga, salgo con amigas al café"

"Quiero volver atrás, que pasen tres meses y volver a empezar con otro grupo"

"No sé lo que quiero, si cultivar (rosas) o no. Estoy bloqueada"

Por falta de tiempo se marcha 30 minutos antes para el autobús

Al final de la sesión se le entregó una Matrix de cierre del grupo para que se llevaran un resumen de la experiencia grupal, que se les propone rellenar antes de cerrar la sesión (Ver figura 1). 


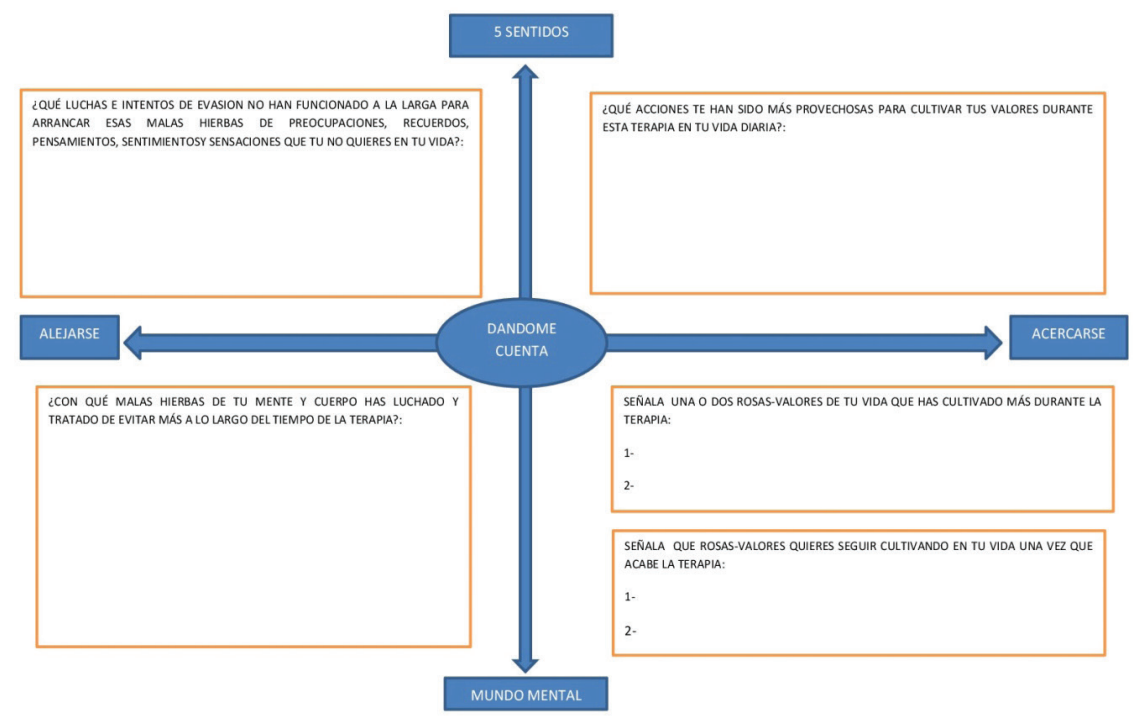

Figura 1. Matrix Final de la Terapia ACT de Grupo.

Sobre la cuestión de las metáforas en la terapia de grupo usamos, como se cita anteriormente, la Metáfora del Jardín de rosas y malas hierbas como metáfora raíz que vertebra toda la terapia de grupo (ver libro Ruiz, 2017). Esta es la metáfora la utilizamos como eje central en la terapia de grupo ACT ya que nuestro objetivo es aumentar la flexibilidad psicológica a través de las discriminaciones entre el control verbal vs el experiencial (contenidos vs procesos), entre la evitación experiencial y la activación conductual comprometida hacia valores y la perspectiva deíctica del yo observador o contexto.

$\mathrm{Al}$ inicio de las sesiones valoramos "las rosas y sus cultivo" (lo que valoran y las acciones comprometidas con esto; y "las malas hierbas y las acciones de evitación experiencial controladas por estas". Para ello usamos el Cuestionario de Experiencias del jardín de la vida-EQGL (Trillo Padilla \& Ruiz Sánchez, 2017) como medida de las rosas y su cultivo en la semana; y el AAQ-II de 7 ítems como medida de la lucha y fusión a las malas hierbas durante la semana.

Igualmente esta metáfora sirve de elemento verbal, visual y experiencial (solemos representarla y dramatizarla en vivo), junto con otros ejercicios experienciales y metáforas para el trabajo de discriminación y como referencia de cómo evolucionan las conductas clínicamente relevantes en el mismo grupo: rosas y acciones comprometidas-CCR2; malas hierbas y evitaciones-CCR1. 


\section{CONCLUSIÓN}

El trabajo con los miembros de los grupos focalizados en la toma de conciencia del darse cuenta de las diferencias (discriminaciones) entre los contenidos vs procesos, evitaciones experienciales vs aproximaciones a acciones valiosas y las perspectivas deícticas del yo observador, son claves para el trabajo de la ACT de grupo.

Utilizar las terapias contextuales, en este caso concreto ACT, en grupo es una experiencia más que enriquece la investigación psicológica y supone un cambio cualitativo para el paciente que se somete a ella. Esta nueva vía de intervención grupal en contextos sanitarios públicos abre una puerta a la investigación basada en la evidencia para el futuro.

\section{REFERENCIAS}

Agevli y cols. (2007).Acceptance and Commitment Therapy Group Therapy Protocol Addictions Intensive Outpatient Program PTSD/Substance Use Dual Diagnosis Program.VA Maryland Health Care System Acceptance and Commitment Therapy (ACT) Team

Alvarez, J., McLean, C., Harris, A. H. S. Rosen, C. S., Ruzek, J., \& Kimerling, R. (2011). The comparative effectiveness of cognitive processing therapy for male veterans treated in a VHA posttraumatic stress disorder residential rehabilitation program. Journal of Consulting and Clinical Psychology, 79, 590-599.

Applebaum, A.J., Lichenthal, W.G., Pessin, H.A., et al. (2012). Factors associated with attrition from a randomized controlled trial of meaning-centered group psychotherapy for patients with advanced cancer. Psycho-Oncology, 21, 1195-204.

Austin, S. F., Sumbundu, A. D., Lykke, J., \&Oestrich, I. H. (2008). Treating panic symptoms within everyday clinical settings: The feasibility of a group cognitive behavioural intervention. Nordic Journal of Psychiatry, 62(4), 287-293.

Barnes-Holmes, D; Hayes, S.C and Diamond, S. (2001). Self and self-directed rules. In S.C. Hayes; D. Barnes-Holmes and B. Roche (eds.). Relational Frame Theory.A post-skinnerian account of human language and cognition (pp. 119-140).Ner York: Kluwer Academic.

Blum, N., St. John, D., Pfohl, B., Stuart, S., McCormick, B., Allen, J., ... Black, D. W. (2008). Systems Training for Emotional Predictability and Problem Solving (STEPPS) for outpatients with borderline personality disorder: A randomized controlled trial and 1-year follow-up. The American Journal of Psychiatry, 165(4), 468-478.

Bohni, M. K., Spindler, H., Arendt, M., Hougaard, E., \& Rosenberg, N. K. (2009).A randomized study of massed three-week cognitive behavioural therapy schedule for panic disorder.ActaPsychiatricaScandinavica, 120(3), 187-195.

Boone, M. S., \& Manning, J. (2012). A pilot study of an Acceptance and Commitment Therapy group for anxiety and depression in a college counselingcenter.Manuscript in preparation. 
Bos, E. H., van Wel, E. B., Appelo, M. T., \&Verbraak, M. J. P. M. (2010). A randomized controlled trial of a Dutch version of Systems Training for Emotional Predictability and Problem Solving for borderline personality disorder. Journal of Nervous and Mental Disease, 198(4), 299-304.

Burlingame, G.M., MacKenzie, K.R. \& Strauss, B. (2004).Small group treatment: Evidence for effectiveness and mechanisms of change. In M. J. Lambert (Ed.), Bergin \& Garfield's Handbook of Psychotherapy and Behavior Change, 5th Ed. New York: Wiley \& Sons, pp. 647-696.

Burlingame, G., Strauss, B. \& Joyce, A (2013). Change mechanisms and effectiveness of small group treatments, In M. J. Lambert (Ed.), Bergin \& Garfield's Handbook of psychotherapy and behavior change, 6th Ed. New York: Wiley \& Sons. (pp. 640-689)

Castilho, P.,Margarida, A.,Viegas, R.,Carvalho, S.,Madeira, N.,andJoão Martins, M. (2015). Preliminary results of an Acceptance and Commitment Therapy (ACT) brief group intervention for psychosis.ARC Publishing.

Chu, B.C., Colognori, D., Weissman, A.S and Bannon, K. (2009).An Initial Description and Pilot of Group Behavioral Activation Therapy for Anxious and Depressed Youth. Cognitive and Behavioral Practice16, (4), Pages 408-419

Clarke,S., Kingston,J., James, K.,Bolderston, H. y Remington, B.(2013).Acceptance and Commitment Therapy group for treatment-resistant participants: A randomized controlled trial. Journal of Contextual Behavioral Science, 3,179-188

Daltry, R.M.2015) A Case Study: An ACT Stress Management Group in a University CounselingCenter, Journal of College Student Psychotherapy, 29(1), 36-43

DiGiuseppe, R., \&Tafrate, R. C. (2003). Anger treatment for adults: A meta-analytic review. Clinical Psychology: Science and Practice, 10, 70-84.

Dimeff, L. A. \&Linehan, M. M. (2008). Dialectical behavior therapy for substance abusers. Addiction Science and Clinical Practice. 4(2), 39-47.

Fleming, J.E. and Kocovsky, N.L. (2014). Mindfulness and Acceptance-Based Group Therapy for Social Anxiety Disorders: A Treatment Manual.

Eilenberg,T., Frostholm,L. and Kronstrand, L. (2014).Acceptance \& Commitment Group Therapy (ACT-G) for severe health anxiety.The research Clinic for Functional Disorders and Psychosomatics, Aarhus University Hospital, Denmark

Fereidooni, S.; Gharaei, B.; Birashk, B.; Sahraeian, A.; Mohammad, S. \& Hosein, D. (2015).Efficacy of Group Behavioral Activation Treatment as Supplemental Treatment to Pharmacotherapy in Inpatients with Depressive Disorders.Journal of Mood Disorders 5(3):104-12

FernándezLiria, A; Rodríguez Vega, B; Muñoz, A y Cebolla, S. (2012).Psicoterapia en el ámbito de la sanidadpública. Factorescomunes y psicoterapia de grupo. En: Desviat, M. y Moreno, A. Acciones de Salud Mental en la Comunidad. Asociación Española de Neuropsiquiatría. Madrid,2012. 
Foody, M., Barnes-Holmes, Y., Barnes-Holmes, D., Törneke, N., Luciano, C., Stewart, I., \&McEnteggart, C. (2014). RFT for clinical use: The example of metaphor. Journal of Contextual Behavioral Science, 3(4), 305-313.

García-Campayo, J., Pascual, A., Alda, M. y Oliván, B. Tratamientopsicológico en el trarstorno de somatización: Eficacia y Propuestas de Intervención. (2005). Revista de Psicopatología y PsicologíaClínica.Volumen 10(1),15-23.

Gómez,M.J. , Luciano, C., Páez-Blarrina, M., Valdivia-Salas, F.J.R y Gil-Luciano, B. (2014). Brief ACT Protocol in At-risk Adolescents with Conduct Disorder and Impulsivity. International Journal of Psychology and Psychological Therapy, 14, 3, 307-332

Guerin, B. (2015). How to Rethink Psychology. Routledge. London and New York.

Guerin, B. (2016). How to Rethink Human Behavior. A Practical Guide To Social Contextual Analysis. Routledge. London and New York.

Guerin, B. (2017).How to Rethink Mental Illness: The Human Contexts Behind the Labels. Routledge. London and New York.

Gutteling, B., Montagne, B., Nijs, M., van des Bosch, L.M.C. (2012). Dialectical behavior therapy: is outpatient group psychotherapy an effective alternative to individual psychotherapy?. Preliminary conclusions. Comprehensive Psychiatry 53. 1161-1163

Hadlandsmyth,K. , White,K. ,Nesin, A.E. y Greco, L. (2013).Proposing an Acceptance and Commitment Therapy Intervention to Promote Improved Diabetes Management in Adolescents: A Treatment Conceptualization. International Journal of Behavioral consultation and Therapy. (7). 4.pp. 12-16.

Harvey, R., Black, D. W., \& Blum, N. (2010). Systems Training for Emotional Predictability and Problem Solving (STEPPS) in the United Kingdom: A preliminary report. Journal of Contemporary Psychotherapy, 40(4), 225-232.

Hayes, S. C. (1984). Making sense of spirituality. Behaviorism, 99-110.

Hayes,S.C. and Jacobson, N.S. (1987).A contextual approach to therapeutic change.En Psychotherapists in clinical practice: Cognitive andbehavioral perspectives, (pp. 327-387). New York: Guilford Press

Hervás,E.S., Gradolí, V.T., Gurrea, R., Bou, N.M. y Gallús, E.M. (2002).Terapiacognitivoconductualbreve en un grupo de dependientes a drogas.Asociación Española de Neuropsicología.

Hoekstra, R. (2008). Functional Analytic Psychotherapy for Interpersonal Process Groups: A Behavioral Application. International Journal of Behavioral Consultation and Therapy $4,2$.

Hopko, D. R., Robertson, S. M. C., \& Colman, L. (2014). Behavioral Activation Therapy for Depressed Cancer Patients: Factors Associated with Treatment Outcome and Attrition. JBCT 4, 4 
Jhonson Norton, M. Evaluation of a Brief Behavioral Activation Therapy for Depression (BATD) Group Protocol in An Inpatient Geriatric Psychiatry Facility. (2010) .Tesis. Tuscaloosa, Alabama

Jónsson, H., \&Hougaard, E. (2009). Group cognitive behavioural therapy for obsessive-compulsive disorder: A systematic review and meta-analysis. ActaPsychiatricaScandinavica, 119(2), 98-106.

Juarascio A ${ }^{1}$, Shaw J, Forman E, Timko CA, Herbert J, Butryn M, Bunnell D, Matteucci A, Lowe M. (2013).Acceptance and commitment therapy as a novel treatment for eating disorders: an initial test of efficacy and mediation. Behavior Modification, 37(4):459-89.

Kanas, N. (1986) Group therapy with schizophrenics: A review of controlled studies. International Journal of Group Psychotherapy 36, 339-351.

Kanas, N. (1996) Group Therapy for Schizophrenic Patients. Washington, DC: American Psychiatric Press.

Lakoff, G., \& Johnson, M. (1980). Metaphors we live by. Chicago, IL: University of Chicago Press.

Leahey, T.H (1998). Historia de la Psicología, principalescorrientes de pensamientopsicológico. Prentice Hall.

Linehan, M. M., Comtois, K. A., Murray, A. M., Brown, M. Z., Gallop, R. J., Heard, H. L., ... Lindenboim, N. (2006). Two-Year Randomized Controlled Trial and Follow-up of Dialectical Behavior Therapy vs Therapy by Experts for Suicidal Behaviors and Borderline Personality Disorder. Archives of General Psychiatry, 63(7), 757-766.

Rajabi, S., \&Yazdkhasti, F. (2014). TheEffectiveness of Acceptance and CommitmentGroupTherapyonAnxiety and Depression in Womenwith MS WhoWereReferredtothe MS Association. Journal of ClinicalPsycology, 6(1), 29-38.

Myler, C. (2012). ACT for Depression and Anxiety Group - Cornell University Counseling and Psychological Services. In Association for Contextual Behavioral Science

Norcross JC, WampoldBE. (2011). What works for whom: tailoring psychotherapy to the person.Journal Clinical Psychology, 67(2):127-132.

Padilla, D. y Jiménez, M.A. (2014).Experienciaclínica de psicoterapiagrupal contextual en adolescentes con problemasemocionales. ClínicaContemporánea, 5. 53-68.

Pichon-Rivière, E. (1987). El procesocreador, ed. Nueva Visión, Buenos Aires.

Pichon-Rivière, E. (1995).Diccionario de Psicología Social, compilacióntemática de sus escritosrealizadaporPichonRivière, Joaquín, y cols., ed. Nueva Visión, Buenos Aires.

Pichon-Rivière, E. (1999). El procesogrupal, ed. Nueva Visión, Buenos Aires.

Polk, K. (2009). The Idiost Guide to ACT in Groups. Manuscrito no publicado.

Polk, K. \&Schoendorff, B. (2014). ACT Matrix. A New Approach to Building Psychological Flexibility Across Setting and Populations. New Harbinger Publications, Inc. 
Polk, K, \&Schoendorff, B. (2016).The Essential Guide to the ACT Matrix: A Step-byStep Approach to Using the ACT Matrix Model in Clinical Practice. New Harbinger Publications, Inc.

Powers, M. B., Sigmarsson, S. R., \&Emmelkamp, P. M. G. (2008). A meta-analytic review of psychological treatments for social anxiety disorder.International Journal of Cognitive Therapy, 1(2), 94-113.

Quismondo, L.L., Ríos, F.L., Pedraza, E.R., Liria, A.F. y Lahera, G. (2016). Effect of an acceptance and commitment group therapy in the treatment of panic disorder and agoraphobia. A preliminary study

Ruiz, J.J; Ruiz, I. y Andujar, P. (2016).Empoderamientopsicológicobasado en ACT y Mindfulness. Lulu.com

Ruiz, J.J (coordinador). (2017).Terapia de Aceptación y Compromiso de Grupo.Basada en la Teoríadel Marco Relacional y el Socioconductismo. Lulu.com

Smith M, Glass G, Miller T. (1980) The benefits of psychotherapy. Baltimore MD: John Hopkins University Press.

Soler, J., Pascual, J. C., Tiana, T., Cebrià, A., Barrachina, J., Campins, M. J., Pérez, V. (2009). Dialectical behaviour therapy skills training compared to standard group therapy in borderline personality disorder: A 3-month randomised controlled clinical trial. Behaviour Research and Therapy, 47(5), 353-358.

Soleimani,M.; Mohammadkhani,P.; Dolatshahi,B., Alizadeh,H.; Overmann,K.; and Coolidge, F.1. (2015). A Comparative Study of Group Behavioral Activation and Cognitive Therapy in Reducing Subsyndromal Anxiety and Depressive Symptoms. Iran J. Psychiatry10:2, 71-78

Soto, S. P., Benerrechea-Arando, L. C., \&Struch, J. M. (2013). Aplicación de la terapia de aceptación y compromiso en pacientes con fibromialgia: una experiencia clínica. Cuadernos de medicina psicosomática y psiquiatría de enlace, (106), 6.

Swain, J., Hancock, K., Dixon, A., Koo, S., \&Bowman, J. (2013). Acceptance and CommitmentTherapyforanxiouschildren and adolescents: studyprotocolfor a randomizedcontrolled trial. Trials, 14(1), 140.

Thibodeau PH y Boroditsky L (2011) Metaphors We Think With: The Role of Metaphor in Reasoning. PLoS ONE 6(2).

Tonarelli, S.B., Pasillas,R., Alvarado,L., Dwivedi, A., and Cancellare, A. (2016). Acceptance and Commitment Therapy Compared to Treatment as Usual in Psychosis: A Systematic Review and Meta-Analysis. Journal Psychiatry, 19, 3.

Torneke, N. (2016). Aprendiendo TMR. Unaintroducción a la Teoríadel Marco Relacional y sus aplicacionesclínicas. Editorial Didacbook S.L. Úbeda.

Treloar, A.S. (2011). Therapeutic Groups with Adult Trauma Survivors. Anita Treloar

Urbano, J. and Love, R. (2011). Dialectical Behavior Therapy Group Skills Training in a Community Mental Health Setting: A Pilot Study. Journal Group Psychotherapy, 61 (4), pp. 645-657 
Vanderberghe, L. (2015). A Functional Analytic Approach to Group Psychotherapy. The Behavior Analyst Today Volume 10, Number 1

Villatte, M., Villatte, J. L., \& Hayes, S. C. (2016). Mastering the clinical conversation: Language as intervention. New York: Guilford Press.

Vinogradov, S. y Yalom, I. (1996).Guíabreve de psicoterapia de grupo.Paidós, Barcelona

Whittingham, K., Sanders, M., McKinlay, L., \&Boyd, R. N. (2014). Interventionsto reduce behavioralproblems in childrenwith cerebral palsy: an RCT. Pediatrics,

Yalom I. (1985).The theory and practice of group psychotherapy. New York: Basic Books. 